\title{
Gold nanoparticle
}

assemblies: Thermal behaviour under optical excitation

\section{Bruno Palpant, ${ }^{1}$ Yannick Guillet, ${ }^{1}$ Majid Rashidi-Huyeh,, ${ }^{1,2}$ and Dominique Prot,,}

${ }^{1}$ Université Pierre et Marie Curie - Paris 6, CNRS UMR 7588, INSP, Campus Boucicaut, 140 rue de Lourmel, Paris, F-75015 France

${ }^{2}$ Department of Physics, University of Sistan and Baluchistan, Zahedan, Iran

${ }^{3}$ Université Paris Sorbonne - Paris 4, France

E-mail: bruno.palpant@upmc.fr

\begin{abstract}
The optical response of materials based on gold nanoparticle assemblies depends on many parameters connected to both material morphology and light excitation characteristics. The optical energy absorbed is then converted into heat through different nanoscale energy exchange mechanisms. This heating subsequently modifies itself the optical properties. We investigate the interplay between the optical and thermal responses of nanocomposite media under its theoretical aspect. In this first paper, the thermal response of gold nanoparticle assemblies under pulsed optical excitation is considered. Both conventional and original modelling approaches are presented. We first underline the role of electromagnetic interactions between particles in a dense assembly in its linear optical response. We then show how the interaction of light with matrix-embedded gold nanoparticles can result in the generation of thermal excitations through different energy exchange mechanisms. Finally, we demonstrate the possible significant influence of the heat carrier ballistic regime and phonon rarefaction in the cooling dynamics of an embedded gold nanoparticle subsequent to ultrafast pulsed laser excitation.
\end{abstract}

The remarkable optical properties of gold nanoparticles associated with the surface plasmon resonance phenomenon have usually been thought to result in concrete developments in the nanophotonic field. However, one cannot but notice that the main recent breakthroughs have rather been achieved in the domain of thermal applications of these optical properties. Indeed, as optical and thermal responses are in fact closely bound, gold nanoparticles can be considered together as nanometric heat sources and probes for local temperature variations via their optical behaviour. The energetic conversion realized by gold nanoparticles which are able to transform at the nanoscale an electromagnetic radiation into heat emitted toward their environment [1] may be indeed relevant in numerous fields. Let us mention some examples. In plasmonic devices [2] local heating may alter the guiding of the electromagnetic wave by gold nanostructures and therefore requires to be well controlled. Gold nanoparticles are also expected to be used in microscopy for labelling biologic cells: Nanoparticle heating by light absorption enables to modify the optical response of their local environment [3]. In the medical area, photothermal cancer therapy based on gold nanoparticles has yet become a very promising technique: assemblies of gold nano-objects (nanorods or dielectric-metal core-shells) absorb light energy transmitted through biologic tissues and transform it into heat which diffuses toward local environment. By using an appropriate targeting method for carrying particles close to affected cells, the latter will be destroyed by overheating [4-6]. One may also take advantage of this local heating around particles for inducing local phase or morphology transformation in the surrounding medium. On the one hand, this can enable the measurement of nanoscale heat transfer through the investigation of such phase transformations [1]. On the other hand, this could be used to modify the global medium optical properties [7]. This effect has been supposed to be at the origin of the optical limitation phenomenon in colloidal solutions (induced light scattering by formation of gas bubbles around gold colloids) [8,9]. Metal nanoparticles are also considered as model defects for studying the damage of optical devices induced by powerful lasers [10-12]. The dynamics of the light-heat conversion in a gold nanoparticle and of the thermal release toward its environment appears then to be a relevant issue in all these domains.

The aim of the present paper is to provide to the reader not only the basic theoretical tools for describing linear optical properties of gold nanoparticle assemblies, but also original approaches developed in our group allowing to describe the dynamics of the optical-thermal energy conversion in such nanocomposite media. The consequences of nanoscale heat transport on the ultrafast relaxation dynamics in nanocomposite media will be highlighted. The results presented here will be used in a forthcoming article to determine the influence of thermal effects on the nonlinear optical response of gold nanoparticle assemblies. 


\section{Linear optical properties of gold nanoparticles: Some elements}

For describing the propagation of an electromagnetic wave in an isotropic and homogeneous medium in the linear regime the usual complex optical index $\tilde{n}=n+i$ א will be considered. $n$ is the refractive index and $\kappa$ the extinction coefficient, proportional to the absorption coefficient $\alpha=4 \pi \kappa / \lambda$ ( $\lambda$ is the wavelength in vacuum). $\tilde{n}$ is linked with the dielectric function $\varepsilon=\varepsilon_{1}+i \varepsilon_{2}$ through $\varepsilon=\tilde{n}^{2}$. In this section, we will give some basic elements regarding the optical response of embedded gold nanoparticles which will be useful for the following.

\subsection{Dielectric function of gold}

The dielectric function of the noble metals has been widely investigated in the past [13-19]. It may include, in the near UV-visible spectral range, the influence of both the quasi-free conduction electrons (sp band) and the bound d ones. Hence, the total dielectric function of noble metals can be written as the sum of the intraband transition contribution (transitions within the conduction band) and the interband one (transitions from $\mathrm{d}$ to $\mathrm{sp}$ bands):

$$
\varepsilon_{m}=\chi^{f}+\varepsilon^{i b} .
$$

\subsubsection{Intraband contribution: Drude model}

The quasi-free electron susceptibility, $\chi^{f}$, is given by the Drude model [20]:

$$
\chi^{f}(\omega)=-\frac{\omega_{p}^{2}}{\omega(\omega+i \Gamma)} .
$$

$\omega_{p}$ is the volume plasma circular frequency and $\Gamma$ is a phenomenological constant accounting for all the collision processes experienced by the conduction electrons, which are considered as independent. The corresponding contributions to $\Gamma$ are then additive (Matthiessen rule). The dominant contribution is the electron-phonon collision one for electron temperatures up to several thousand Kelvin. In the case of a metal nanoparticle, an additional contribution has to be considered due to electron scattering at the particle surface, which is inversely proportional to particle size [21].

\subsubsection{Interband contribution: Rosei's model}

Due to Pauli principle interband transitions present an energy threshold corresponding to the excitation of electrons from the top of the $\mathrm{d}$ band to states just above the Fermi level $\left(E_{F}\right)$ in the conduction band. Thus, below this threshold, $\varepsilon_{2}^{i b}=0$. For gold, this threshold lies in the visible (at $\sim 2.4 \mathrm{eV}$ ) [16], which explains its colour in the bulk state. Contrarily to the intraband contribution to the dielectric function, the interband one cannot be evaluated through a classical approach. Indeed, it is necessary to sum over all possible transitions from an occupied state in the $d$ band to an unoccupied state in the sp band. The detailed band structure has then to be modelled [13,22-25]. This point has been treated in the 1970s by Rosei and coworkers [ 23-26].

\section{Key points for the calculation}

We describe here the procedure which is usually employed to get the modification of interband transitions. One determines the imaginary part of $\varepsilon^{i b}$ first, the real one being subsequently deduced by Kramers-Kronig analysis. In the framework of Lindhard's theory, $\varepsilon_{2}^{i b}$ can be written as [20,24]:

$\varepsilon_{2}^{i b}(\omega)=\frac{K}{\omega^{2}} \sum_{i, j} \int_{B Z}\left|M_{i \rightarrow j}(\mathbf{k})\right|^{2}\left[f_{i}(\mathbf{k})-f_{j}(\mathbf{k})\right] \delta\left[\omega_{i j}(\mathbf{k})-\omega\right] d \mathbf{k} .(3)$

where $K$ is a constant factor, $\hbar \omega_{i j}=E_{j}-E_{i}$ is the energy gap between initial $i$ and final $j$ states, $\mathbf{k}$ is the electron wave vector, $M_{i \rightarrow j}(\mathbf{k})$ is the matrix element between states $i$ and $j$, and $f_{n}(\mathbf{k})$ is the occupation factor of state $n$. The photon wave vector being negligible, the transition is quasi-vertical which imposes energy conservation accounted for by the Dirac function in Eq. (3). Integration is performed over the Brillouin zone (BZ). In gold, the points $X$ (onset at $\sim 1.9 \mathrm{eV}$ $[16,26]$ ) and especially $L$ (onset at $\sim 2.4 \mathrm{eV}[26]$ ) of the BZ provide the main contributions to $d \rightarrow s p$ interband transitions in the visible. Around these two points the $M_{d \rightarrow s p}(\mathbf{k})$ can be considered as locally constant, since the wave number range matching the energy conservation condition is very narrow $[23,26]$. Thus, for a transition around $X$ or $L, \varepsilon_{2}^{i b}$ is proportional to the joint density of states (i.e. associated with a transition of given energy) which is linked with the densities of states in the $d$ and sp bands and accounts for the energy and wave vector conservation [23]:

$J_{d \rightarrow s p}^{X, L}(\omega)=\int_{X, L} \frac{2}{(2 \pi)^{3}}\left[f_{d}(\mathbf{k})-f_{s p}(\mathbf{k})\right] \delta\left[\omega_{d-s p}(\mathbf{k})-\omega\right] d \mathbf{k}$.

Eq. (3) can then be transformed into:

$\varepsilon_{2}^{i b}(\omega)=\frac{K}{\omega^{2}}\left(\left|M_{d \rightarrow s p}^{X}\right|^{2} J_{d \rightarrow s p}^{X}(\omega)+\left|M_{d \rightarrow s p}^{L}\right|^{2} J_{d \rightarrow s p}^{L}(\omega)\right)$.

d-band states being fully occupied, Eq. (4) can be simplified since $f_{d}=1$ whatever $\mathbf{k}$. The conduction band density of states, $f_{s p}$, can be determined by solving the Boltzmann equation as will be shown below (§ 3.1.1). The conduction band being isotropic [20], the integration over wave vector is replaced by the equivalent integration over energy. One finally calculates $J_{d \rightarrow s p}^{X, L}(\omega)$ which depends on the band structure characteristics, as energies $E_{d}$ and $E_{s p}$ and effective mass $m_{\text {eff }}$, around the BZ points involved. Let us underline that when one aims at determining the modification of $\varepsilon^{i b}$ in gold induced by the absorption of an ultrafast laser pulse, the only significant contribution is that of transitions near point $L$.

\section{Band structure model}

The band structure of gold has been studied at the beginning of the 1970s [27]. The $\mathrm{d}$ band group lies a few eV below $E_{F}$. The sp band crosses $E_{F}$ in the vicinity of the points $X, L$ and $\Sigma$ of the BZ. For a given direction of the reciprocal space, it 
can locally be described by a parabolic branch scheme (quasifree electron behaviour) around the points contributing to the optical response, $X$ and $L$, the dispersion law being then given as $E(\mathbf{k})=\hbar^{2} \mathbf{k}^{2} / 2 m_{\text {eff }}$ [26].

\subsection{Linear optical response of a single gold nanoparticle}

When reducing the volume available for conduction electrons in a metal particle until its size becomes much smaller than the light wavelength, the well-known surface plasmon resonance (SPR) phenomenon arises. Indeed, electrons experience the same homogeneous electromagnetic field and respond collectively (from a quantum point of view, it corresponds to the coherent excitation of electronic transitions within the conduction band). This remarkable property also appears in the expression of the extinction cross section of a metal sphere given by the Mie theory at the dipolar electric order [28]. At resonance the amplitude of the local electric field in the particle, $\mathbf{E}_{\ell}$, is enhanced as compared to the one of the applied field, $\mathbf{E}_{0}$. In other words, the modulus of the complex local field factor $f_{\ell}=E_{\ell} / E_{0}$ can be greater than one at the SPR. Numerous recent technological developments in various fields like nonlinear optics [29], sensors, or biomedical imaging are based on this property. In a very simple approach consisting in solving Laplace equation for an isolated metal sphere in an infinite medium one gets for $f_{\ell}$ :

$$
f_{\ell}=\frac{3 \varepsilon_{d}}{\varepsilon_{m}+2 \varepsilon_{d}},
$$

where $\varepsilon_{d}$ is the dielectric function of the surrounding medium. The above equation reveals that $\mid f_{\ell}$ exhibits a resonant behaviour as $\left|\varepsilon_{m}+2 \varepsilon_{d}\right|$ reaches a minimum value. As the existence of such a resonant behaviour can be explained by simple electromagnetic considerations using macroscopic parameters as dielectric functions, the origin of the local field enhancement is often known as dielectric confinement.

In gold and copper nanoparticles the SPR is located in the visible and presents a lower oscillator strength than in silver, for which the SPR peaks in the near UV. This stems from the significant coupling, in the former case, between bound and quasi-free electron transitions. In addition, the larger the host medium refractive index, the higher the SPR oscillator strength and the lower its frequency. Details regarding the SPR characteristics in noble metal nanoparticles and nanocomposite media can be found in several books $[21,30,31]$. Let us just point out that the quantum finite size effects (electronic confinement) have a large influence on the gold nanoparticle optical response and have been the subject of many theoretical and experimental investigations [21,32-34].

\subsection{The case of nanoparticle assemblies}

\subsubsection{Different theoretical approaches}

The approach presented above is well suited for an isolated particle or, in a pinch, for diluted nanocomposite media. In a medium consisting of an assembly of metal nanoparticles spread in a dielectric host the "ensemble effects" have to be taken into account as soon as the density of nanoparticles reaches a sufficient value. Metal volume fraction, $p$, is then the relevant parameter along with the spatial arrangement of the particles. With increasing $p$ mean field effects appear. Further rising $p$ then renders electromagnetic interactions between neighbouring particles significant. A large number of effective medium theories have been developed in order to account for such effects. Each one is adapted to a specific material morphology and a specific concentration range. They generally provide an analytical description of the whole medium effective optical response through its effective dielectric function, given the different individual constituent ones. The pioneering and most known one is the MaxwellGarnett theory (MGT) [35], which supposes no interaction between metal spheres and is thus limited to weakly concentrated media, typically $p<\sim 13 \%$ at the SPR (the precise $p$ range for its validity actually depends on wavelength).

Effective medium theories allow the modelling of the electromagnetic response of an inhomogeneous medium while keeping the formalism appropriate to homogeneous media. However, the hypotheses they impose regarding the shape and size of the particles, their distance, spatial arrangement, concentration and environment in the medium, restrict their validity or lead to develop more and more complex models. An alternative approach can be considered thanks to high power numerical computing possibilities. Given a virtual sample with finite volume, one solves numerically the equations describing mater-radiation interaction in this volume, as Maxwell equations or generalized Mie theory, in order to determine the local electromagnetic field topography. The virtual sample may be chosen as to statistically represent the real global medium. The spatial mean value and dispersion of the field can then be extracted from the results.

We have used such a method to calculate the local field topography in assemblies of spherical gold nanoparticles randomly spread in a transparent host medium. Particle size is supposed to be much lower than the wavelength. This method is based on a multiple-scattering model initially developed for the case of ensembles of scattering spheres, in the formalism of the recursive transfer matrix (T-matrix) [36]. The virtual sample is exposed to an external plane wave in the steady regime with linear polarization. The field in a particle is then linked to the applied field and the sum of all fields scattered by the other particles through the associated T matrix [37]. The field scattering by each sphere is treated using Mie theory where the maximum multipolar order of the development, $n_{\max }$, is imposed. The stability and convergence of the calculation are ensured by the choice of this maximum order, which is as high as the particles are close together and of large size. The dielectric functions of the gold spheres and host medium are assimilated to the ones of the bulk state. The results of these calculations are of course linked with the optical response; for instance, the absorption coefficient of 
the whole medium is proportional to the mean square of the local field modulus. We will also see further that the latter is involved at the fourth power in the effective third-order susceptibility of nanocomposite materials.

\subsubsection{Influence of gold concentration}

The MGT predicts a red-shift of the SPR with increasing $p$ [21]. This is the consequence of the mean field effect (the MGT does not take into account interactions between particles). One also observes an increase of the SPR absorption band magnitude simply due to the increase of metal amount, the dielectric medium being transparent. We have recently analyzed through simple cases the role of particle interactions on the local field properties depending on the particle arrangement and applied field polarization. For this, the multiple-scattering approach described above has been used. To summarize, the alignment of particles along the applied field direction results in the strengthening and redshift of the resonance in each particle, whereas an alignment perpendicular to $\mathbf{E}_{\mathbf{0}}$ leads to the decrease of the SPR oscillator strength and a slight blue-shift. These changes increase with reducing inter-particle distance. Their mutual influence acts on both the amplitude and phase of $\mathbf{E}_{\ell}$. The results obtained in these simple cases, the details of which will be published elsewhere, allow us to analyze more complex situations, describing real samples. We have thus applied our method to the electromagnetic response of a silica host where about 50 to 60 spherical gold nanoparticles, $2 \mathrm{~nm}$ radius, are randomly spread. For this, we have generated distributions at a given $p$ value, ensuring that the minimum inter-particle distance is compatible with the convergence of the calculation, the value of $n_{\max }=3$ being imposed as to keep a reasonable computing time. Figure 1 presents the calculated data for two distributions, one (a) having a weak concentration $(p=1 \%)$, the other (b) a high one ( $p=22 \%)$. The virtual samples are also shown at the same scale in the respective inserts. The quantity reported is the spectral variation of the relative local field intensity $\left(\left.\sim f_{\ell}(\lambda)\right|^{2}\right)$ averaged in each particle of the distribution, along with its mean value in the sample. For evaluating this mean value the particles located at the edge of the sample (corresponding to about 30\% of the total sample volume) have been disregarded, since their local environment does not match the one the particles in a real sample would have.

One can observe on Fig. 1 the much larger dispersion of the spectra for the larger $p$ value. The presence, in the latter case, of a few very intense resonance bands shifted to longer wavelengths is actually due to a group of very close particles in the virtual sample. Such a behaviour has already been highlighted in bidimensional semi-continuous gold films (existence of hot spots for the local field) [39-41]. The redshift of the SPR maximum with increasing $p$ is retrieved for the mean spectrum. One can also notice that in such random distributions there seem to be no noticeable amplification of the mean field intensity; this could stem from the homogeneous distribution of the inter-particle axis orientation relative to the applied field polarization, which should be confirmed by further investigations. It is worth noticing, however, that the medium third-order optical nonlinear response, which is sensitive to the product $f_{\ell}^{2}\left|f_{\ell}\right|^{2}$ [38], may exhibit a volume mean value larger than in the case of an isolated particle. One can finally notice on Fig. 1 that the reducing of the interparticle distance associated with the increase of $p$ leads to the asymmetric broadening of the mean SPR band, absorption being enhanced in its red tail. This fact stems from the increased occurrence of dense particle packing as the one already discussed in Fig. 1(b), and is then fully ascribable to interactions between neighbouring particles.
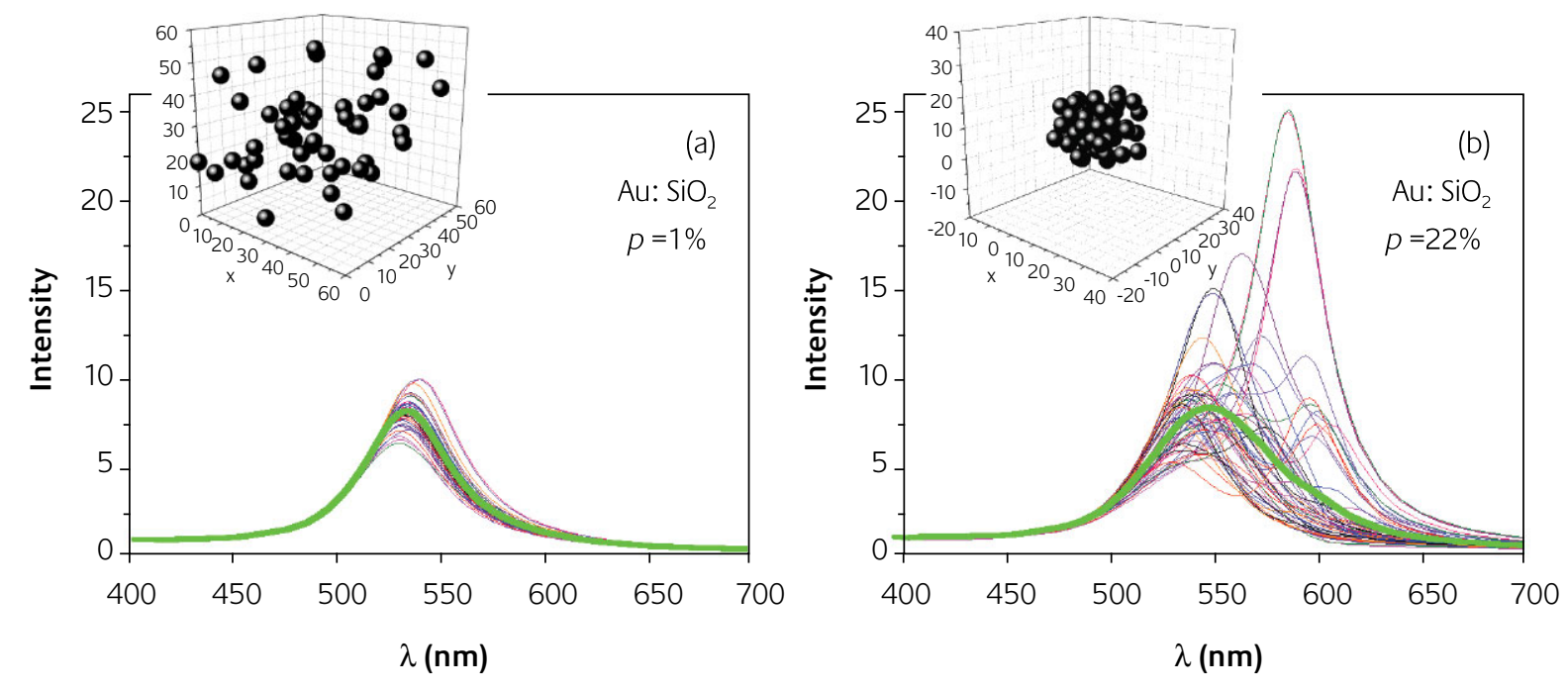

Figure 1

Relative local field intensity averaged in each particle, (thin colored curves), and mean value in the whole sample corrected from edge effects (thick green curve) for a random distribution (insert) of 53 gold nanoparticles (2 nm radius) in a $\mathrm{SiO}_{2}$ matrix, with metal concentration $1 \%$ (a) and $22 \%$ (b). 


\section{Dynamics of thermal exchanges in nanocomposite media under pulsed laser excitation}

We have been working for a few years on the development of models aiming at describing the dynamics of thermal exchanges in nanocomposite media under pulsed laser excitation depending on pulse duration and energy, particle environment and metal concentration - by accounting for the relevant physical mechanisms involved. Here, we present the main different approaches and the most recent developments.

The response of a nanocomposite medium to a laser pulse is ruled by a series of different mechanisms, each exhibiting its own dynamics [42-52]: light energy absorption by electrons, redistribution within the conduction electron gas through electron-electron collisions, relaxation toward metal lattice by electron-phonon scattering, and then particle cooling down by heat transfer to the surrounding medium. Note that the short time domain of the relaxation - the first few picoseconds after excitation - has been widely investigated by several groups $[42,45,48,50]$. We will only give here some basic elements regarding our approaches and disregard any further refinement.

\subsubsection{Athermal regime: Resolution of the Boltzmann equation}

For sake of simplicity, we consider an excitation at photon energy lower than the IB transition threshold. In the opposite case, one should include the effect of Landau damping. When a metal at temperature $T_{0}$ absorbs an ultrashort light pulse through transitions within the conduction electron gas, the electron distribution is driven out of equilibrium. Internal thermalization ensured by electron-electron collisions leads to a Fermi-Dirac profile at temperature $T_{e}>T_{0}$, while electronphonon scattering converts energy into heat within the particle, the temperature $T_{l}$ of which rises. Note that the notion of temperature in its strict statistical definition, in a metal object smaller than the heat carrier mean free path, may be questionable. $T_{l}$ will rather be considered as a measure of thermal internal energy. The athermal regime corresponds to the phase during which the electron distribution is off equilibrium. It lasts for a few hundred femtoseconds. It has of course no reason to be considered in a slow excitation regime where electrons and phonons are always at thermodynamic equilibrium.

In the athermal regime, the relevant quantity for describing metal properties is the electron distribution function, $f(E, t)$, where $E$ is electron energy. Its time dependence is ruled by Boltzmann equation which writes:

$$
\frac{\partial f(E, t)}{\partial t}=\left.\frac{\partial f(E, t)}{\partial t}\right|_{\text {source }}+\left.\frac{\partial f(E, t)}{\partial t}\right|_{e-e}+\left.\frac{\partial f(E, t)}{\partial t}\right|_{e-p h} \text { (7) }
$$

Particles being smaller than the wave penetration depth, the excitation can be considered as homogeneous within the electron gas. Electron diffusion is then ignored in Eq. (7). Moreover, we neglect the influence of the surrounding matrix.
This remains justified at short delays after pulse absorption inasmuch as there is no chemical-type interaction at the interface.

In the right part, the first term depicts the evolution of $f$ due to light pulse absorption (source term), while the last two ones denote the variation rate of $f$ due to electron-electron and electron-phonon collisions, respectively. These last few years several research groups have developed and carried out more or less refined methods allowing to solve Boltzmann equation in order to describe the athermal regime in noble metals [53-59]. We have elaborated an approach partly similar to the one previously reported in Refs. [57-59]. It is based on a dual-time relaxation approximation for e-ph scattering and the Landau theory of Fermi liquids for the e-e one.

\section{Source term}

Dependent on time and electron energy $\varepsilon=E-E_{F}$, the source term is proportional to the instantaneous power absorbed by metal volume units, $P_{a b s}(t)$, as well as to the instantaneous variation of the occupation rate at given $\varepsilon$. This second factor is evaluated by calculating the probability for an electron to be promoted at $\varepsilon$ by absorbing a photon $\hbar \omega$, while subtracting its probability of being promoted at $\varepsilon+\hbar \omega$. The proportionality factor is then determined by imposing the total energy conservation.

\section{Electron-electron scattering}

This term originates from both the vanishing of electrons of excess energy $\varepsilon$ and the creation of electrons at this energy resulting from screened Coulomb scattering with other electrons. In the weak perturbation case the Landau theory of Fermi liquids allows to apply the time relaxation approximation for describing the first contribution [60]. The corresponding electron lifetime varies as $\tau_{e-e}=\tau_{0} E_{F}{ }^{2} / \varepsilon^{2}$. The closer the energy to $E_{F}$, the weaker the e-e scattering probability, which is a direct consequence of Pauli principle. $\tau_{0}$ represents the lifetime the electron would have in the absence of this exclusion principle. It is evaluated by considering it as a fitting parameter of the model and comparing with results of relaxation dynamics measurements reported in the literature. Its value then ranges within $0.3-1.0$ fs. The second contribution to e-e scattering is calculated from already defined quantities through the expression established by Richie [61].

\section{Electron-phonon scattering}

The e-ph scattering term in Eq. (7) stems from spontaneous emission, stimulated emission and absorption of phonons. In the last two cases the scattering rate is proportional to the number of available states in the reservoir (phonon population of the reservoir following a Bose-Einstein distribution at $T_{l}$ ). The e-ph scattering term is thus split into two contributions, each treated in the frame of the time relaxation approximation. Both depend on the energy transfer rate from the electron gas to the phonon bath, $\dot{q}$. The second one also depends on 
the number of phonons created until time $t$ and the mean value of the phonon energy (given by the Debye model for the density of states). A nondimensional factor, S, accounts for the relative weight of both emission processes and absorption.

\section{Implementation}

Boltzmann equation is solved numerically by using a finite difference method with a 1 fs time step and a 5 meV energy step. $\tau_{0}, \dot{q}$ and $\mathrm{S}$ are free parameters fitted on experimental data from the literature $[52,54]$. This procedure is rendered possible as these three parameters are independent of $\varepsilon$ and $t$, and as they act on the dynamics in distinct timescales. The typical duration of a calculation of the relaxation dynamics over 10 ps is lower than 5 minutes with a personal computer.

\section{Finite size effects}

The characteristics of the scattering processes driving the evolution of the electron distribution can be modified by finite size effects in nanoscale metal objects. One globally observes the reduction of the e-e and e-ph collision time with decreasing $R_{p}$ [62-64]. One of the origins of this behaviour lies in the reduction of the screening of the electron-electron and electron-ion coulomb interactions. The increase of the e-ph scattering rate is also ascribed to the modification of the metal phonon spectrum due to the appearance of vibration modes inherent to confinement [65]. These finite size effects result in a modification of parameters $\tau_{0}$ and $\dot{q}$.

\section{Conclusion about the athermal regime}

The resolution of Boltzmann equation allows to account for the athermal regime for the electron distribution and its consequences on the dynamics of the optical response at short time scale after excitation by an ultrashort laser pulse. We will show in a forthcoming article an example of such a calculation and its application to the calculation of the hot electron contribution to the third-order nonlinear response of nanocomposite materials. The model used, however, is valid inasmuch as thermal exchanges between particles and their environment remain negligible. When the conduction electron gas has reached internal equilibrium - that is, when it can be described by a Fermi-Dirac distribution at temperature $T_{e}$ - one enters the thermal regime. It is then possible to include the influence of the environment in a three-temperature model.

\subsubsection{Thermal regime: Three-temperature model}

Exchanges between electrons and phonons within metal particles (two-temperature model, 2TM) are very often considered alone to describe the ultrafast optical response dynamics. And yet the presence of the surrounding medium begins to play a significant role just after the first few picoseconds following the excitation, and then cannot be neglected. Moreover, the 2TM does not allow to deal with long-lasting pulse regimes. On the contrary, the threetemperature model (3TM) that we present here enables in principle to carry out the calculation of the dynamics whatever the pulse duration. As the description of this model has already been partly published [66,67], we just recall here the main elements in order to explain the foundation of the last section.

\section{Hypotheses}

For sake of clarity, the conduction electron gas is considered as instantaneously thermalized, i.e. the finite duration of the athermal regime is neglected. This assumption is justified for an ultrashort pulsed excitation of high energy for which the duration of this regime is short, and of course for long pulsed excitation with which the electron gas is always at thermodynamic equilibrium with the metal lattice. It is nevertheless possible to connect the approaches developed for each regime through a modified 3TM, which is out of the scope of this paper.

In the 3TM the thermodynamic characteristics of the different media are likened to the ones of their bulk phase. It would be relatively easy to include in a phenomenological way some effects linked with confinement. The metal/ dielectric contact is supposed to be perfect and a possible interface thermal resistance is disregarded. The near field radiative heat transfer is neglected, which is valid in the case of metal nanoparticles the size of which is limited to a few tens nanometers.

\section{Coupled equations}

Let us consider a nanoparticle with radius $R_{p}$ isolated in a dielectric host medium. This particle absorbs homogeneously a part of the energy carried by an incident light pulse. The equations to be solved describe the energetic exchanges between the conduction electron gas at temperature $T_{e}(t)$, metal lattice at temperature $T_{l}(t)$ and surrounding matrix at temperature $T_{m}(\mathbf{r}, t)[67,68]$. The input excitation (source term for the electron gas) is described through $P_{a b s}(t)$, defined above and depending on pulse characteristics, material absorption and metal concentration. The first equation then depicts the electron energy evolution due to both this source term and the electron-phonon scattering:

$$
C_{e} \frac{\partial T_{e}}{\partial t}=-G\left(T_{e}-T_{l}\right)+P_{a b s}(t)
$$

$C_{e}=\gamma_{e} T_{e}$ is the electron gas specific heat, $\gamma_{e}$ is a constant depending on metal, $G$ is the e-ph coupling constant. The second equation accounts for the evolution of the lattice thermal energy, fed by the e-ph coupling and transferred to the surrounding matrix through the interface:

$$
C_{l} \frac{\partial T_{l}}{\partial t}=G\left(T_{e}-T_{l}\right)-\frac{H(t)}{V} .
$$

$C_{l}$ is the metal lattice heat capacity, $V$ is the particle volume and $H(t)$ is the instantaneous power released from particle to matrix. Let us now focus on the evaluation of this last term. 
It has to be treated in a different way depending on the excitation and observation conditions. One has to consider the heat transport mechanisms in the dielectric medium from the interface. In the general case, at small space and time scales, this transport is described by Boltzmann equation or a simplified version; this will be developed later. As soon as the observation timescale (and/or the space scale) is large as compared with phonon lifetime $\tau$ (resp. mean free path $\Lambda$ ) the use of Fourier's law may nonetheless be considered as reasonable for describing thermal transport. $\tau_{p h}$ and $\Lambda$ are worth, for two usual dielectric media, $\tau_{p h} \sim 130$ fs and $\Lambda \sim 0.5$ $\mathrm{nm}$ in silica $\left(\mathrm{SiO}_{2}\right)$ and $\tau_{p h} \sim 850$ fs and $\Lambda \sim 5.4 \mathrm{~nm}$ in alumina $\left(\mathrm{Al}_{2} \mathrm{O}_{3}\right)$.

\section{The case of classical diffusive heat transport: Fourier's law}

One adds to Eqs. (8) and (9) the conventional parabolic law of heat diffusion for describing conduction in the dielectric matrix:

$$
\frac{\partial T_{m}}{\partial t}=\frac{\kappa_{m}}{C_{m}} \Delta T_{m}
$$

where $C_{m}$ and $\kappa_{m}$ are the matrix specific heat and thermal conductivity, respectively. Assuming that $T_{l}(t)=T_{m}\left(R_{p}, t\right)$, the function $H(t)$ simply writes:

$$
H(t)=\left.S \kappa_{m} \frac{\partial T_{m}}{\partial r}\right|_{r=R},
$$

where $S$ is the particle surface.

\section{Influence of metal concentration}

This model, initially suited for an isolated particle and then for a dilute nanocomposite medium, has been extended to the case of dense assemblies of nanoparticles [67]. Indeed, by preserving spherical symmetry allowing to treat in a relatively

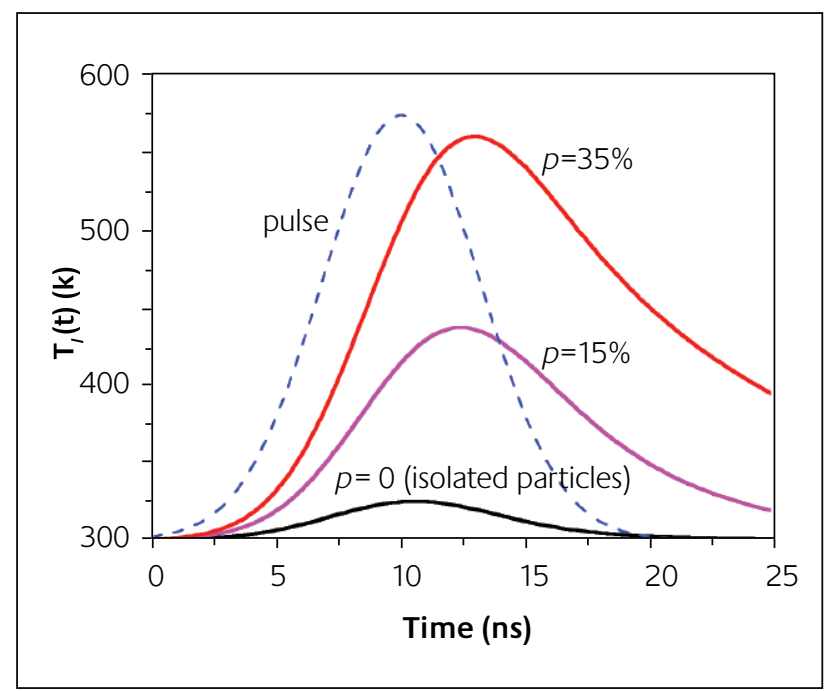

\section{Figure 2}

Temperature dynamics of gold nanoparticles $\left(R_{p}=1.3 \mathrm{~nm}\right)$ in silica under pulsed laser excitation (pulsewidth: $7.5 \mathrm{~ns}$, absorbed peak power: $5 \times 10^{17} \mathrm{~W} \mathrm{~m}^{-3}$ ) for three metal concentrations. The pulse profile is reported in blue (arbitrary units). easy way the coupled equations and keeping the values of both metal concentration and particle size, we have included the influence of thermal exchanges between neighbouring particles in the medium. The use of Fourier's law now imposes the additional condition that the distance between particles be large as compared with $\Lambda$. We have shown that metal concentration plays via these exchanges a crucial role in the temperature dynamics in the case of long pulsewidth excitation, as well as at long pump-probe delays in the case of ultrashort excitation. The main origin of such a dependence lies in the fact that when the heat front emitted by a particle reaches a neighbouring one, the temperature gradient at the surface of the latter decreases, which results in the slowing down of its cooling. This is illustrated in the nanosecond excitation regime on Fig. 2 which presents the dynamics of the particle temperature for three different concentrations. One can obviously observe the large increase of the $T_{l}$ peak value as well as the time shift of this peak with increasing $p$. These results will be used later for evaluating the consequences of particle heating in the optical Kerr response of nanocomposite materials.

\section{The case of ballistic-diffusive heat transport}

For very short times, small distances and then high metal concentrations, or for matrices with high thermal conductivity, Fourier's law is no longer valid. The Boltzmann transport equation (BTE) then has to be invoked. The question of thermal transport at small time and space scales has been dealt with by several authors [69-75]. An alternative solution to the difficult implementation of the BTE, so called ballisticdiffusive equations (BDE) and based on the time relaxation approximation, has been proposed by Chen [71,74]. It is particularly suited for the study of transient thermal phenomena at the nanoscale, for which it has given results quantitatively similar to the BTE ones while requiring much simplified calculations. It consists in splitting in every point of the medium the heat flux $\mathbf{q}(\mathbf{r}, t)$ and the internal energy $u(\mathbf{r}, t)$ into two distinct parts: $\mathbf{q}=\mathbf{q}_{b}+\mathbf{q}_{d}$ and $u=u_{b}+u_{d}$. The first one depicts the contribution of ballistic phonons emitted from boundaries and the second one accounts for phonons stemming from scattering processes (or re-emitted after absorption) from other points of the medium.

Chen has mainly adapted this method to the case of a thin film undergoing a sudden temperature rise on one of its sides [72]. In our case, we have included the BDE in the 3TM [76]. For this, the term in Eq. (9) describing the energy exchange at particle-matrix interface is calculated as:

$$
H(t)=\int_{S} \mathbf{q}(\mathbf{r}, t) \cdot \mathbf{n} d s .
$$

Flux integration runs over the whole particle surface, and $\mathbf{n}$ denotes the unit vector normal to the latter, pointing toward the dielectric. By writing the heat flux as the integral of phonon intensity over all energies and all directions of space and developing the diffusive contribution to this intensity as a truncated sum of spherical harmonics to order 1 (method 
P1, cf. Ref. [75]), and by considering both the energy conservation law and the BTE in the time relaxation approximation for the intensity, Chen has deduced a set of equations (BDE) after averaging conductivity and phonon mean free path over all frequencies [72]. We do not recall his whole reasoning but just write the main equation he obtains:

$$
\tau_{p h} \frac{\partial^{2} u_{d}}{\partial t^{2}}+\frac{\partial u_{d}}{\partial t}=\nabla \cdot\left(\frac{\kappa_{m}}{C_{m}} \nabla u_{d}\right)-\nabla \cdot \mathbf{q}_{b},
$$

where $\tau_{p h}$ is the average phonon lifetime. This equation differs from the Cattaneo-Vernotte one (i.e. the hyperbolic diffusion equation obtained by adding an inertial term to Fourier's law as to account for the finite carrier lifetime) from the additional last term relative to ballistic processes. Chen has shown the crucial significance of this term at small space and time scales. He has also highlighted the relevance of using nondimensional parameters for flux, energy, time and space coordinates.

\subsubsection{Consequences of the nanoscale heat conduction in the host medium}

We have implemented the BDE into our 3TM in the case of a spherical metal-dielectric core-shell nanoparticle. This work has been carried out together with S. Volz from the EM2C laboratory, CNRS, Ecole Centrale Paris, France. A gold core $\left(R_{p}=10 \mathrm{~nm}\right)$ is surrounded by an alumina shell the thickness of which, $d$, is of the same order of magnitude as the phonon mean free path. This particle absorbs (through electron gas excitation) the energy of a 110 fs Gaussian pulse with peak power $P_{a b s 0}=1.4 \times 10^{21} \mathrm{~W} \mathrm{~m}^{-3}$. The internal boundary of the dielectric shell (at $r=R_{p}$ ) then emits ballistic phonons at particle lattice temperature, $T_{l}(t)$. The BDE along with appropriate boundary and initial conditions are solved together by a numerical explicit finite difference scheme. Figures 3 and 4 present the result obtained with $d=\Lambda=5.4 \mathrm{~nm}$ in the thermalizing and adiabatic boundary conditions, respectively. The first one corresponds to a full thermalization (temperature $T_{0}$ imposed at the outer surface) while the second corresponds to a full isolation (cancellation of the heat flux at the outer surface). The quantity reported on these figures is the time evolution of the relative temperature variation of both electron gas and metal lattice. Initial equilibrium temperature is worth $T_{0}=300 \mathrm{~K}$. The results provided by the Fourier law have been added for comparison.

In both cases $T_{e}$ increases very rapidly up to $2200 \mathrm{~K}$ at the pulse end. At short times after excitation $(t<5 \mathrm{ps})$ energy relaxation is dominated by electron-phonon scattering in metal and remains insensitive to external heat transfer, which explains that electron temperature does not depend on the heat transport mechanism. On the contrary, lattice temperature, which is much lower than $T_{e}$ due to the much larger specific heat of the phonon bath as compared with the electron gas one, is sensitive to the account for the ballistic regime from the very beginning. After the first picoseconds,

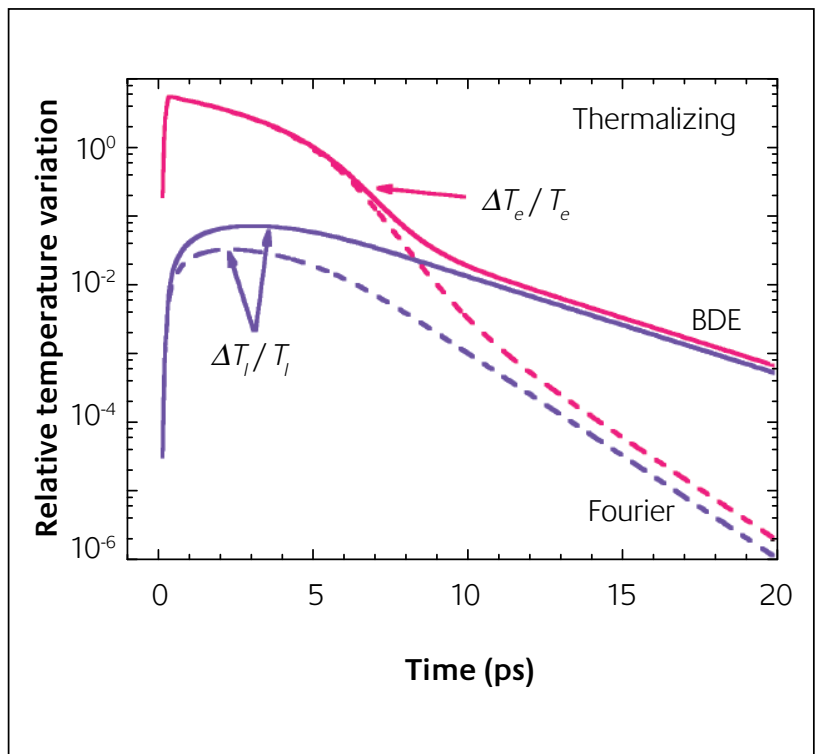

Figure 3

Dynamics of the electron and lattice relative temperature variations induced in a spherical gold nanoparticle (radius: $10 \mathrm{~nm}$ ) surrounded by an alumina shell (thickness: $5.4 \mathrm{~nm}$ ) by an ultrashort laser pulse (pulsewidth: 110 fs). Data are obtained using the ballistic-diffusive approximation (solid lines) and Fourier's law (dashed lines). The equilibrium temperature is $T_{0}=300 \mathrm{~K}$. The boundary condition imposes $T_{0}$ at the shell outer surface (thermalizing condition). (Collaboration with S.Volz, EM2C laboratory, CNRS, Ecole Centrale Paris, France).

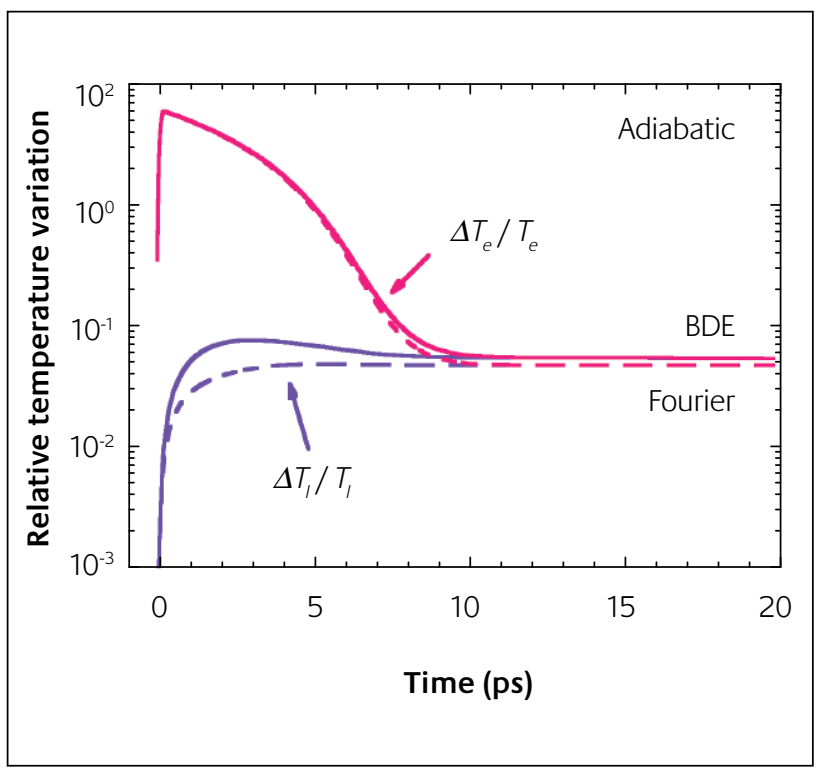

Figure 4

Same as Fig. 3 but the boundary condition consists now in imposing the cancellation of the heat flux at the shell outer surface (adiabatic condition).

electron temperature also exhibits a strong dependence on the heat transport mechanism in the dielectric shell. As can be seen on Fig. 3, in the thermalizing boundary case electrons and phonons are at quasi-equilibrium after about $11 \mathrm{ps}$. The $\mathrm{BDE}$ predicts from then a much slower relaxation than 
Fourier's law, which is due to phonon rarefaction at metal/ dielectric interface. In other words, Fourier's parabolic law overestimates the possible energy redistribution channels due to its inherent assumption of the existence of diffusive processes at any point of the medium and at any time. Moreover, as the input energy remains for a longer time in the particle, the value of $T_{l}$ predicted by the BDE is higher than the one evaluated with Fourier's law. We have additionally shown that shell thickness has a great influence on the relaxation dynamics [76].

The case of adiabatic boundary conditions (Fig. 4) is quite different since, as the shell thickness is as small as the phonon mean free path, the dielectric medium is very rapidly isothermal due to the homogeneous redistribution of the total input energy within the core-shell nanoparticle. $T_{e}$ and $T_{l}$ then reach a plateau after $\sim 10$ ps only. A slight difference between the BDE and Fourier's law predictions can nevertheless be still observed, especially for $T_{l}$ in the first instants after excitation.

In a real experimental situation the relaxation is likely to exhibit an intermediate behaviour between these two extreme boundary condition cases, depending on the conductivity of the medium surrounding the core-shell particle. Beyond, the results presented here suggest that thermal transport in the matrix may play a crucial role in the cooling dynamics of ultrafast heated gold nanoparticles and then in the optical response of nanocomposite media.

\section{Conclusion}

In spite of the apparent simplicity of the surface plasmon resonance phenomenon, the analysis of the optical response of gold nanoparticle assemblies is far from being straightforward. Indeed, it depends on many parameters regarding both material morphology and excitation characteristics. We have investigated the interplay between the optical and thermal responses of such media. We have willfully limited our presentation to theoretical approaches, either commonly used, or recently developed in our group, enabling to deal with different situations and including most of the time ensemble effects which can be significant in dense nanoparticle assemblies. We have shown how the interaction of light with matrix-embedded gold nanoparticles can result in the generation of thermal excitations through different energy exchange mechanisms. Considering the possible inadequacy of conventional macroscopic approaches for describing heat conduction at small time and space scales, we have been able to demonstrate the influence of the heat carrier ballistic regime and phonon rarefaction in the cooling dynamics of an embedded gold nanoparticle subsequent to ultrafast pulsed laser excitation. In a forthcoming paper we will describe how thermal processes can affect the optical response of a nanoparticle assembly. We will then connect both aspects and point out their involvement in the nonlinear optical response of nanocomposite media.

\section{Acknowledgements}

The financial support of the Agence Nationale de la Recherche (program ANR/PNANO 2006, project EThNA) is gratefully acknowledged.

\section{About the authors}

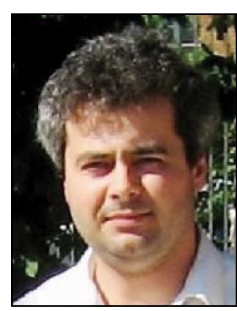

Bruno Palpant is associate professor at the Université Pierre et Marie Curie - Paris 6. He conducts his research activities in the Institut des NanoSciences de Paris where he is in charge of the group for nonlinear optics and thermal effects in metal nanoparticles. He got his PhD in 1998 from University of Lyon (France) about quantum size effects in matrix-embedded noble metal clusters, before joining Keio University (Japan) for one year. He has been interested in the linear and nonlinear optical response of noble metal nanoparticles and more recently in their link with thermal conduction at small space and time scales.

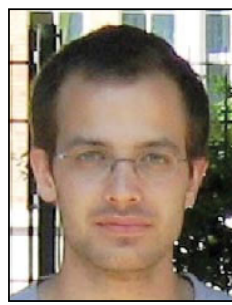

Yannick Guillet got his $\mathrm{PhD}$ in Université Pierre et Marie Curie - Paris 6 in 2007, on the ultrafast dynamics of the nonlinear response of gold nanoparticle assemblies. Henow develops femtosecond laser experiments as a post-doctoral researcher.

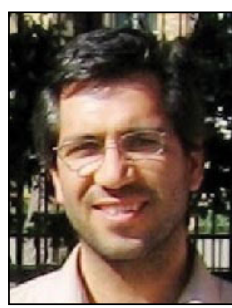

Majid Rashidi-Huyeh is associate professor at University of Sistan and Baluchistan, Zahedan (Iran). He achieved his PhD in 2006 about the influence of thermal effects on the optical response of metal-dielectric nanocomposite materials.

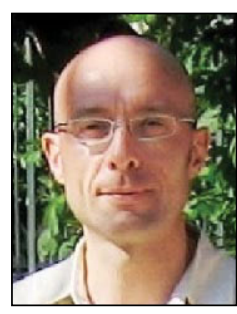

Dominique Prot is associate professor of physics at the Université Paris Sorbonne - Paris IV. His research is conducted at the Institut des Nanosciences de Paris (Université Pierre et Marie Curie - Paris 6) on electromagnetic properties of nanocomposite materials and is mainly focused on electric field calculation in matrix-embedded metal nanoparticles.

\section{References}

1 A.O. Govorov and H.H. Richardson, Nanotoday, 2007, 2 (1), 30

2 S.A. Maier, M.L. Brongersma, P.G. Kik, S. Meltzer, A.A.G. Requicha, and H.A. Atwater, Adv. Mater., 2001, 13, 1501

3 S. Berciaud, L. Cognet, G.A. Blab and B. Lounis, Phys. Rev. Lett., 2004, 93 , 257402 
4 L.R. Hirsch, R.J. Stafford, J.A. Bankson, S.R. Sershen, B. Rivera, R.E. Price, J.D. Hazle, N.J. Halas and J.L. West, PNAS, 2003, 100, 13549

5 C. Loo, A. Lin, L. Hirsch, M. H. Lee, J. Barton, N. Halas, J. West and R. Drezek, Technol. Cancer Res. Treat., 2004, 3, 33

6 P.K. Jain, I.H. El-Sayed and M.A. El-Sayed, Nanotoday, 2007, 2 (1), 18

7 M. Maaza, O. Nemraoui, C. Sella and A.C. Beye, Gold Bull., 2005, 38, 100

8 R.G. Ispasoiu, L. Balogh, O.P. Varnavski, D.A. Tomalia and T. Goodson, III, J. Am. Chem. Soc., 2000, 122, 11005

9 L. François, M. Mostafavi, J. Belloni and J.A. Delaire, Phys. Chem. Chem. Phys., 2001, 3, 4965

10 S. Papernov and A.W. Schmid, J. Appl. Phys., 2002, 92, 5720

11 F. Bonneau, P. Combis, J.L. Rullier, M. Commandré, A. During, J.Y. Natoli, M.J. Pellin, M.R. Savina, E. Cottancin and M. Pellarin, Appl. Phys. Lett., 2003, 83, 3855

12 B. Bertussi, J.-Y. Natoli, M. Commandré, J.-L. Rullier, F. Bonneau, P. Combis and P. Bouchut, Opt. Commun., 2005, 254, 299

13 H. Ehrenreich and H.R. Philipp, Phys. Rev., 1962, 128, 1622

14 M.L. Thèye, Phys. Rev. B, 1970, 2, 3060

15 P.B. Johnson and R.W. Christy, Phys. Rev. B, 1972, 6, 4370

16 D.E. Aspnes, E. Kinsbron, and D.D. Bacon, Phys. Rev. B, 1980, 21, 3290

17 R.A. Innes and J.R. Sambles, J. Phys. F: Metal Phys., 1987, 17, 277

18 D.J. Nash and J.R. Sambles, J. Mod. Opt., 1996, 43, 81

19 Handbook of Optical Constants of Solids, Vols. I and II, edited by E.D. Palik (Academic Press, New York, 1985/1991)

20 N.W. Ashcroft and N.D. Mermin, Solid State Physics (Saunders College Pub., 1976)

21 U. Kreibig and M. Vollmer, Optical Properties of Metal Clusters (Springer Verlag, Berlin Heidelberg, 1995)

22 B.R. Cooper, H. Ehrenreich and H.R. Philipp, Phys. Rev., 1965, 138, A494.

23 R. Rosei and D.W. Lynch, Phys. Rev. B, 1972, 5, 3883

24 R. Rosei, Phys. Rev. B, 1974, 10, 474

25 R. Rosei, C.H. Culp and J.H. Weaver, Phys. Rev. B, 1974, 10, 484

26 M. Guerrisi, R. Rosei and P. Winsemius, Phys. Rev. B, 1975, 12, 557

27 N.E. Christensen and B.O. Serphin, Phys. Rev. B, 1971, 4, 3321

28 G. Mie, Ann. Phys. (Leibzig), 1908, 25, 377

29 B. Palpant, in Nonlinear optical properties of matter: From molecules to condensed phases, Series: Challenges and Advances in Computational Chemistry and Physics, Vol. 1, pp. 461-508, edited by M.G. Papadopoulos, J. Leszczynski and A.J. Sadlej (Springer, 2006)

30 C.F. Bohren and D.P. Huffman, Absorption and Scattering of Light by Small Particles (Wiley, New York, 1983)

31 M. Born and E. Wolf, Principles of Optics (Cambridge U. Press, Cambridge, 1999)

32 A. Kawabata and R. Kubo, J. Phys. Soc. Jpn., 1966, 21, 1765

33 C. Yannouleas and R.A. Broglia, Ann. Phys. N.Y., 1992, 217, 105

34 B. Palpant, B. Prével, J. Lermé, E. Cottancin, M. Pellarin, M. Treilleux, A. Perez, J.L. Vialle and M. Broyer, Phys. Rev. B, 1998, 57, 1963

35 J.C. Maxwell-Garnett, Philos. Trans. R. Soc. London, 1904, 203, 385

36 B. Stout, J.C. Auger and J. Lafait, J. Mod. Opt., 2002, 49 (13), 2129

37 D. Prot, D.B. Stout, J. Lafait, N. Pinçon, B. Palpant and S. Debrus, J. Opt. A: Pure and Appl. Opt., 2002, 4, 599.

38 F. Hache, D. Ricard, C. Flytzanis and U. Kreibig, Appl. Phys. A, 1988, 47, 347

39 S. Grésillon, L. Aigouy, A.C. Boccara, J.C. Rivoal, X. Quelin, C. Desmarest, P. Gadenne, V.A. Shubin, A.K. Sarychev and V.M. Shalaev, Phys. Rev. Lett., 1999, 82, 4520
40 V.M. Shalaev and A.K. Sarychev, Phys. Rev. B, 1998, 57, 13265

41 P. Gadenne, X. Quelin, S. Ducourtieux, S. Gresillon, L. Aigouy, J.C. Rivoal, V. Shalaev and A. Sarychev, Physica B, 2000, 279, 52

42 J.-Y. Bigot, J.-C. Merle, O. Cregut and A. Daunois, Phys. Rev. Lett., 1995, 75, 4702

43 Y. Hamanaka, N. Hayashi, S. Omi and A. Nakamura, J. Lumin., 1997, 76\&77, 221

44 M. Nisoli, S. Stagira, S. De Silvestri, A. Stella, P. Tognini, P. Cheyssac and R. Kofman, Phys. Rev. Lett., 1997, 78, 3575

45 M. Perner, P. Bost, U. Becker, M. Mennig, M. Schmitt and H. Schmidt, Phys. Rev. Lett., 1997, 78, 2192; G.V. Hartland, J.H. Hodak, and I. Martini, Comment, ibid, 1999, 82, 3188; M. Perner, G. von Plessen and J. Feldmann, Reply, ibid, 1999, 82, 3189

46 H. Inouye, K. Tanaka, I. Tanahashi and K. Hirao, Phys. Rev. B, 1998, 57, 11334

47 H. Inouye, K. Tanaka, I. Tanahashi, and K. Hirao, Jpn. J. Appl. Phys., 1998, 37, L1520

48 S. Link and M.A. El-Sayed, J. Phys. Chem. B, 1999, 103 (40), 8410

49 J.-Y. Bigot, V. Halté, J.-C. Merle and A. Daunois, Chem. Phys., 2000, 251, 181

50 N. Del Fatti and F. Vallée, Appl. Phys. B, 2001, 73, 383

51 Y. Hamanaka, J. Kuwabata, I. Tanahashi, S. Omi and A. Nakamuka, Phys. Rev. B, 2001, 63, 104302

52 C. Voisin, N. Del Fatti, D. Christofilos and F. Vallée, J. Phys. Chem. B, 2001, 105,2264

53 W.S. Fann, R. Storz, H.W.K. Tom, and J. Bokor, Phys. Rev. B, 1992, 46, 13592

54 C.-K. Sun, F. Vallée, L. Acioli, E.P. Ippen and J.G. Fujimoto, Phys. Rev. B, 1993, 48, 12365; ibid, 1994, 50, 15337

55 R.H.M. Groeneveld, R. Sprik and A. Lagendijk, Phys. Rev. B, 1995, 51, 11433

56 N. Del Fatti, R. Bouffanais, F. Vallée and C. Flytzanis, Phys. Rev. Lett., 1998, $\mathbf{8 1 , 9 2 2}$

57 G. Tas and H.J. Maris, Phys. Rev. B, 1994, 49, 15046

58 C. Suárez, W.E. Bron, and T. Juhasz, Phys. Rev. Lett., 1995, 75, 4536

59 V.E. Gusev and O.B. Wright, Phys. Rev. B, 1998, 57, 2878

60 D. Pines and P. Nozières, The theory of quantum liquids, Vol. I : Normal Fermi liquids (W.A. Benjamin Inc., New York, 1966)

61 R.H. Ritchie, J. Appl. Phys., 1966, 37, 2276

62 C. Voisin, D. Christofilos, N. Del Fatti, F. Vallée, B. Prével, E. Cottancin, J. Lermé, M. Pellarin and M. Broyer, Phys. Rev. Lett., 2000, 85, 2200.

63 A. Arbouet, C. Voisin, D. Christofilos, P. Langot, N. Del Fatti, F. Vallée, J. Lermé, G. Celep, E. Cottancin, M. Gaudry, M. Pellarin, M. Broyer, M. Maillard, M.-P. Pileni and M. Treguer, Phys. Rev. Lett., 2003, 90, 177401

64 J. Lermé, G. Celep, M. Broyer, E. Cottancin, M. Pellarin, A. Arbouet, D. Christofilos, C. Guillon, P. Langot, N. Del Fatti and F. Vallée, Eur. Phys. J.D, 2005, 34, 199

65 E.D. Belotkii, S.N. Luk'yanets and P.M. Tomchuk, Sov. Phys. JETP, 1992, 74, 88

66 B. Palpant, D. Prot, A.-S. Mouketou-Missono, M. Rashidi-Huyeh, C. Sella and S. Debrus, Proc. of SPIE, 2003, 5221, 14

67 M. Rashidi-Huyeh and B. Palpant, J. Appl. Phys., 2004, 96, 4475

68 Y. Hamanaka, J. Kuwabata, I. Tanahashi, S. Omi and A. Nakamuka, Phys. Rev. B, 2001, 63, 104302

69 A.A. Joshi and A. Majumdar, J. Appl. Phys., 1993, 74, 31

70 G. Chen, J. Heat Transfer, 1996, 118, 539

71 G. Chen, Phys. Rev. Lett., 2001, 86, 2297 
G. Chen, J. Heat Transfer ASME, 2002, 124, 320

73 D.G. Cahill, W.K. Ford, K.E. Goodson, G.D. Mahan, A. Majumdar, H.J. Maris, R. Merlin and S.R. Phillpot, J. Appl. Phys., 2003, 93, 793

74 G. Chen, D. Borca-Tasciuc and R. Yang, Nanoscale Heat Transfer, Encyclopedia of Nanoscience and Nanotechnology Vol. X, pp. 1-30, ed. par H.S. Nalwa (Am. Sci. Publ., 2004)

75 Microscale and Nanoscale Heat Transfer, Series: Topics in Applied Physics, Vol. 107, edited by S. Volz (Springer, 2007)

76 M. Rashidi-Huyeh, S. Volz and B. Palpant, J. Heat Transf. ASME (to be published) 